\title{
Approche de l'interaction laser UV - cible liquide par ombroscopie $\mathbf{X}$
}

C. Cachoncinlle, I. Challine, F. Jobard, E. Robert et J.M. Pouvesle

GREMI, CNRS/Université d'Orléans, BP. 6759, 45067 Orléans cedex 2, France

\begin{abstract}
In this work, we used shadowgraphy technics to follow the dynamics of the interaction between a UV excimer laser ( $\mathrm{XeCl}$ at $308 \mathrm{~nm}$ ) and liquid targets (mercury and water drops). These studies have been achieved together with spectral and temporal analysis of the resulting plasma plume. Shadowgraphy of the fragmentation (mercury drop) or evaporation (water drop) were recorded at different times of the energy transfer process.
\end{abstract}

\section{INTRODUCTION.}

La dynamique des processus engendrés par l'interaction d'un laser UV avec une cible liquide a pu être étudier par ombroscopie $X$ grâce à une puissante source $X$ impulsionnelle. Les processus physiques qui accompagnent le transfert d'énergie sont différents selon la nature, métallique ou diélectrique, de la cible.

\section{DISPOSITIF EXPERIMENTAL.}

Le schéma du dispositif expérimental est présenté figure 1. Il comprend principalement un laser UV et flash X synchronisés entre eux. Le laser à excimère de gaz rare XeCl (Lambda Physik EMG $101 \mathrm{MSC}$ ) délivre à $308 \mathrm{~nm}$ une énergie de $200 \mathrm{~mJ}$ par tir. La durée de l'impulsion est de 10 ns. Le flash [1] émet des impulsions de photons $X$ d'énergie comprise entre quelques $\mathrm{keV}$ et quelques dizaines de $\mathrm{keV}$. La dose de rayons $\mathrm{X}$ produite à la fenêtre de sortie de ce flash peut atteindre 1 rad par tir. Cette dose est suffisante pour impressionner un film photographique situé à $1 \mathrm{~m}$ de la source $X$. Cette source a une taille inférieure à $200 \mu \mathrm{m}$. La durée de l'impulsion est de $20 \mathrm{~ns}$. Une photodiode sensible à la fois au rayon $X$ produits par le flash $X$ et aux photons UV de l'impulsion laser permet de contrôler la synchronisation des deux appareils. Le faisceau laser est focalisé sur une cible liquide constituée soit d'une goutte de mercure (cible métallique) soit d'une goutte d'eau (cible diélectrique). Au point d'impact, la densité d'énergie est d'environ de $1 \mathrm{GW} / \mathrm{cm}^{2}$. L'ombre de la goutte (ombroscopie X) est enregistrée sur un film photographique Kodak à différents instants après l'interaction lasercible. Le délai entre l'impulsion laser et l'impulsion de photons $X$ peut être ajustée entre quelques nanosecondes et quelques millisecondes 


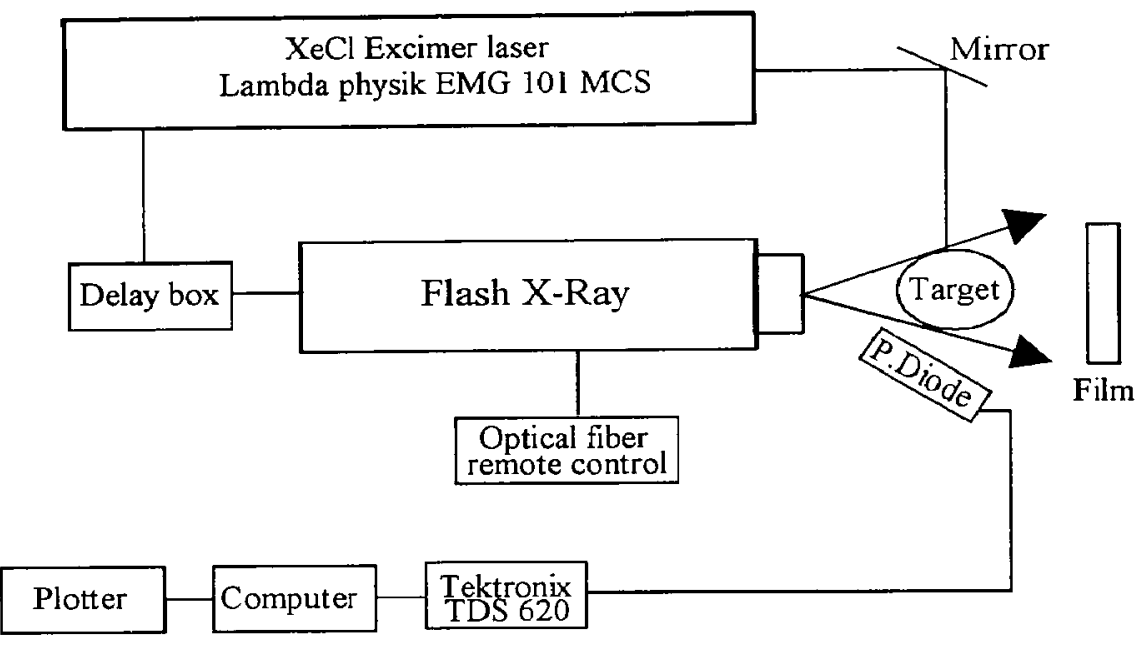

Figure 1: dispositif expérimental.

\section{RESULTATS.}

\section{1 Goutte de mercure.}

Nous avons focalisé l'impulsion laser sur une goutte de métal liquide (mercure) dans l'air. Pendant les premières dizaines de nanosecondes, l'énergie transportée dans le faisceau permet de créer un plasma de mercure au voisinage immédiat de la surface du liquide [2]. L'observation de ce plasma en spectroscopie d'émission montre un continu de radiation fortement décalé vers l'UV traduisant un plasma de température électronique élevée. Puis pendant les quelques centaines de nanosecondes correspondant à la relaxation du plasma, le spectre présente des raies spectrales atomiques et ioniques. A ce stade une grande partie de l'énergie est encore localisée au voisinage de la surface d'interaction. Le transfert final de l'énergie à la matière se fait alors par un effet de piston aucours duquel la surpression engendrée par le plasma laser appuie localement sur la surface du liquide cédant ainsi son énergie sous forme cinétique à différentes gouttelettes de mercure qui sont alors éjectées. Nous avons enregistré par ombroscopie $\mathrm{X}$ cette projection de matière aux instants $\mathrm{t}=10,50,400,640,800$ et $1000 \mu$ s après l'interaction.

\subsection{Goutte d'eau.}

La même expérience a été réalisée sur une goutte d'eau. Dans ce cas, le faisceau pénètre dans la goutte. L'énergie libérée dans ce volume cause alors l'évaporation complète de la goutte. L'ombroscopie $X$ réalisée à différents instants après le tir laser fixe les différentes phases de cette évaporation. Aucun changement notable n'apparaît avant quelques microsecondes. Puis, un gradient de densité commence à apparaître montrant une zone de plus forte densité répartie en périphérie de la goutte et une zone de plus faible densité au centre. Cette inhomogènéité précède le phénomène d'évaporation qui se produit pendant les quelques dizaines de microsecondes suivantes. L'éjection des vapeurs se fait dans la direction du faisceau incident. La goutte est totalement évaporée après une centaine de microsecondes.

\section{Références:}

[1] J.M. Pouvesle et al, Rev. Sci. Instrum. 64 (1993) 2320.

[2] M. Von Allmen; Laser Beam interactions with materials, Springer Verlag, Serie in Materials Science, Berling Heidelberg 1987. 